\title{
DISEÑO DE UNA PMO BASADA EN PMI APLICADA EN UNA UNIVERSIDAD CHILENA
}

\author{
Desing of a PMO based on PMI applied in a Chilean university
}

EPISTEMUS

ISSN: 2007-8196 (electrónico)

ISSN: 2007-4530 (impresa)

Esteban Toledo Pérez de Arce 1

Luis Alvarado Acuña ${ }^{2}$

Jocelyn Castillo Vega ${ }^{3}$

Rocio Veas Mercado 4

Recibido: 20 de septiembre de 2018,

Aceptado: 30 de noviembre de 2018

Autor de Correspondencia:

Rocio Veas Mercado

Correo:rvm014@alumnos.ucn.cl

\section{Resumen}

El presente artículo sintetiza el diseño de una PMO basada en el estándar PMI para la gestión de proyectos. Primeramente, se realiza una adaptación del plan estratégico para la Sede Vallenar, para luego, utilizar el modelo de madurez organizacional y recomendar una PMO apropiada a diseñar, la cual tiene como fin el mejorar las prácticas en dirección de proyectos al generar iniciativas de asesorías, capacitaciones y apoyo a los miembros de la organización para alcanzar un nivel de lenguaje común en dirección de proyectos. Para llevar a cabo este cometido, el diseño de la PMO se apoya en la implementación de la estrategia organizacional a través de la gestión de portafolio al alinear los proyectos con la estrategia institucional y en la materialización de las políticas organizacionales a través de la gestión de programas que agruparán los proyectos generados en la Sede Vallenar de la Universidad de Atacama. Finalmente se obtiene como resultado un nivel de madurez inferior al nivel 1 , recomendando iniciar con capacitaciones en vocabulario técnico.

Palabras clave: PMO, Gestión del Capital Intelectual, Gestión Estratégica, Modelos de Madurez Organizacional en Dirección de Proyectos, Gestión de Portafolio y Gestión de Programas.

\section{Abstract}

This article synthesizes the design of a PMO based on the PMI standard for project management. Firstly, an adaptation of the strategic plan for the Vallenar Headquarters is made, and then, using the organizational maturity model, it is possible to recommend an appropriate PMO to be designed, which aims to improve the practices in the direction of projects by generating consulting initiatives, training and support to the members of the organization to initially reach a level of common language in project management. To carry out this task, the design of the PMO is based on the implementation of the organizational strategy through portfolio management, aligning the projects with the institutional strategy and the materialization of the organizational policies through the management of programs that will group the projects generated in the Vallenar Headquarters of the University of Atacama. Finally, the result is a level of maturity below level 1, recommending starting with technical vocabulary training

Keywords: PMO, Intellectual Capital Management, Strategic Management, Organizational Maturity Models in Project Management, Portfolio Management and Programs Management.

1 Depto. Gestión de la Const., U. Católica del Norte. Av. Angamos 0610, Antofagasta, Chile. Correo: Toledo.perezdearce@gmail.com

2 Depto. Gestión de la Const., U. Católica del Norte. Av. Angamos 0610, Antofagasta, Chile. Correo: lualvar@ucn.cl

3 Depto. Gestión de la Const., U. Católica del Norte. Av. Angamos 0610, Antofagasta, Chile. Correo: Jocelyn.castillov@gmail.com

4 Depto. Gestión de la Const., U. Católica del Norte. Av. Angamos 0610, Antofagasta, Chile. Correo: Rvm014@alumnos.ucn.cl 


\section{INTRODUCCIÓN}

Los proyectos son actividades organizadas, las cuales tienen un inicio y un fin bien definidos, con el fin de crear o asegurar valor, lo cual permite proyectarse en el tiempo, al crecer, desarrollarse y consolidarse para dar cumplimiento a su misión y visión de una organización. La Sede Vallenar de la Universidad de Atacama es una institución de educación superior, ubicada en la ciudad de Vallenar, III Región, Chile, la cual tiene como objetivo principal entregar educación a personas que se acercan a la vida universitaria con una expectativa de desarrollo personal y profesional.

Esta universidad busca desarrollar líneas de investigación de acuerdo a su plan estratégico institucional, las cuales son logrables a través de la adjudicación de proyectos internos de la Universidad y externos como FNDR, FONIS, PCI Conicyt, EXPLORA, entre otros. Estas adjudicaciones impactan en el desarrollo de la organización ya que son fuente de financiamiento, generador de publicaciones y desarrollo investigativo institucional. Localmente, la organización no cuenta con un ente que se encargue de asesorar, guiar y capacitar, en un lenguaje común de gestión de proyectos, es por ello, que se identifica la necesidad de diseñar una PMO la cual sea el ente encargado de integrar metodologías, técnicas, gestionar las lecciones aprendidas y capitalizar la experiencia de la institución, con la finalidad generar mayores ventajas competitivas sostenibles en el tiempo.

La pregunta que se planteada es ¿Cómo se puede mejorar la calidad de los proyectos generados en la Sede Vallenar de la UDA para que cumplan con las expectativas de la Casa Central y de los diversas entidades públicas y privadas para adjudicarse fondos concursables, promover la investigación y generar material de publicación?

Cómo objetivos específicos de la investigación se plantean los siguientes:

- Diseñar una PMO para mejorar el desempeño de la Sede Vallenar en relación a la gestión de los proyectos, que integre los esfuerzos de control de gestión en el alineamiento con los objetivos estratégicos de la institución, la experiencia organizacional, la gestión del conocimiento y las lecciones aprendidas.

- Desarrollar un análisis estratégico, un análisis de madurez organizacional en la institución, para recomendar una PMO apropiada al nivel de desarrollo de la institución en estos ámbitos.

- Presentar un protocolo para capitalizar la experiencia de la organización, capacitar en un lenguaje común y presentar una metodología estándar a utilizar en la generación de proyectos.

\section{METODOLOGÍA}

La metodología es de tipo descriptivo, ya que a partir de las hipótesis planteadas y su comprobación se pretende llegar a un conocimiento explicativo. La metodología de trabajo seguida en la investigación consta de tres etapas principales: definición y diseño de la investigación; preparación, recolección y análisis de la evidencia; y por último conclusiones. (Ver Figura 1).

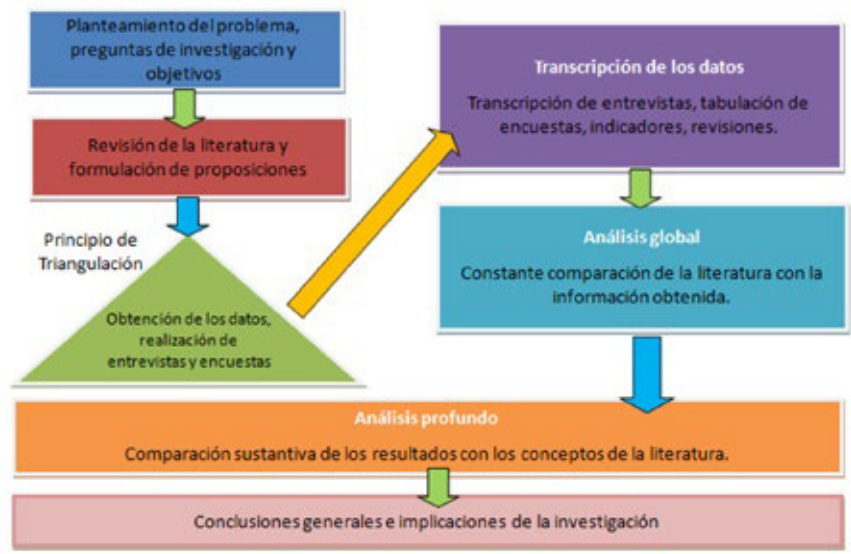

Figura 1. Esquema general de los procesos del Método del caso propuesto para la investigación.

(Fuente: Toledo, 2017 y Shaw, 1999)

Para la Investigación se aplicará el método del caso simple acoplado, donde el estudio se desarrolla en el contexto de educación superior, utilizando cuatro unidades de análisis. (Ver Figura 2).

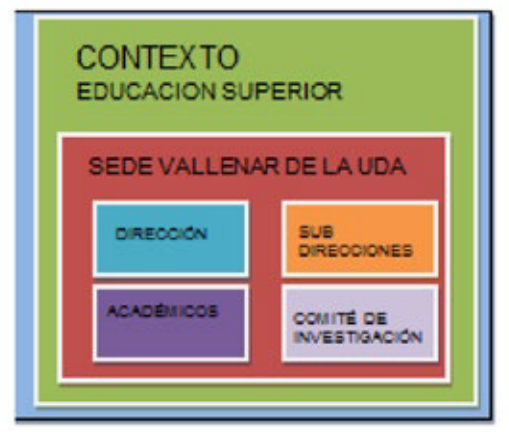

Figura 2. Esquema del método del caso acoplado. (Fuente: Toledo 2017 y Alvarado, 2004)

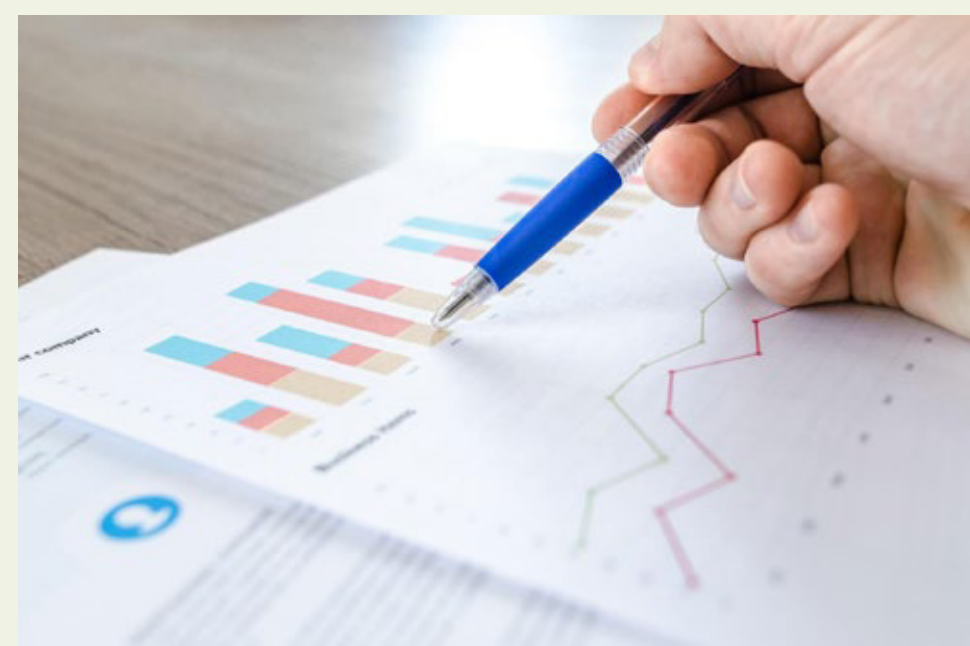




\section{MARCO TEÓRICO}

El marco teórico se divide en los ámbitos generales mostrados en el cuadro sinóptico de la Figura 3:

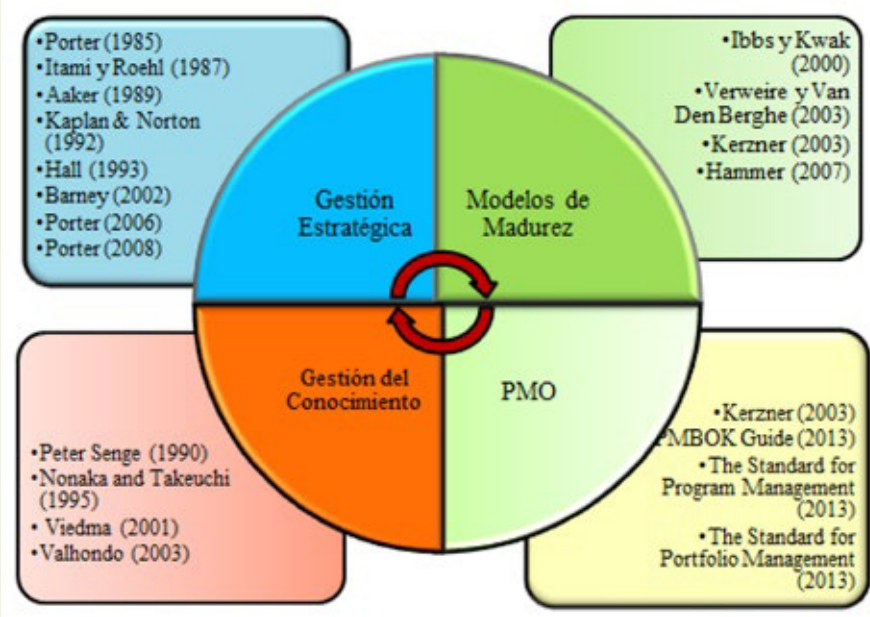

Figura 3. Cuadro Sinóptico de las Teorías Utilizadas

(Fuente: Toledo 2017 y Alvarado, 2004)

- La gestión estratégica en las organizaciones: Es la capacidad de generar cambios positivos en el rumbo de la organización. Se basa en un conjunto de teorías y prácticas que permiten obtener una visión actual interna y externa de la organización. Esta revisión de la organización se conoce como análisis estratégico. Las principales teorías de análisis estratégico son la Teoría Estructural y la Teoría de Recursos y Capacidades.

- Los modelos de madurez organizacional: Todas las organizaciones marchan a través de un proceso de madurez. La curva de aprendizaje de la madurez se mide con el paso de los años (Kerzner, 2001). PMI define un modelo de madurez, como un marco referencial conceptual que define niveles de madurez en ciertas áreas de interés.

- La oficina de dirección de proyectos (PMO): Se puede considerar como una organización de esfuerzos centralizados para agrupar, entregar, capacitar y fomentar las mejores prácticas utilizadas en la gestión de proyectos. Todo esto con el fin de aumentar el éxito en los proyectos y el control de gestión.

- La gestión del conocimiento en las organizaciones: Nonaka y Takeuchi (1995) afirman que entender el proceso de creación de conocimiento organizacional, es preciso primeramente entender la naturaleza del conocimiento, para lograr esto, se debe dividir el conocimiento en dos dimensiones, la ontológica y la epistemológica.

- Gestión del capital intelectual: Viedma (2001), define el capital intelectual como "el conocimiento que produce valor o como el conocimiento que puede convertirse en beneficios. En esta definición se asume que no todo el conocimiento es capital intelectual, sino que solamente el conocimiento que sea selectivo, esencial y estratégico que está estrechamente relacionado con la generación de valor y de beneficios".

\section{Hipótesis}

- Hipótesis de Segundo Grado № 1: Aplicando los modelos para medir el nivel de madurez de la organización, se puede lograr un incremento en el nivel de desarrollo de la organización y por ende una mejora en la gestión de sus proyectos.

- Hipótesis de Segundo Grado № 2: Diseñando una PMO se puede lograr proporcionar una gestión de proyectos eficaz con un plan detallado y coordinado,

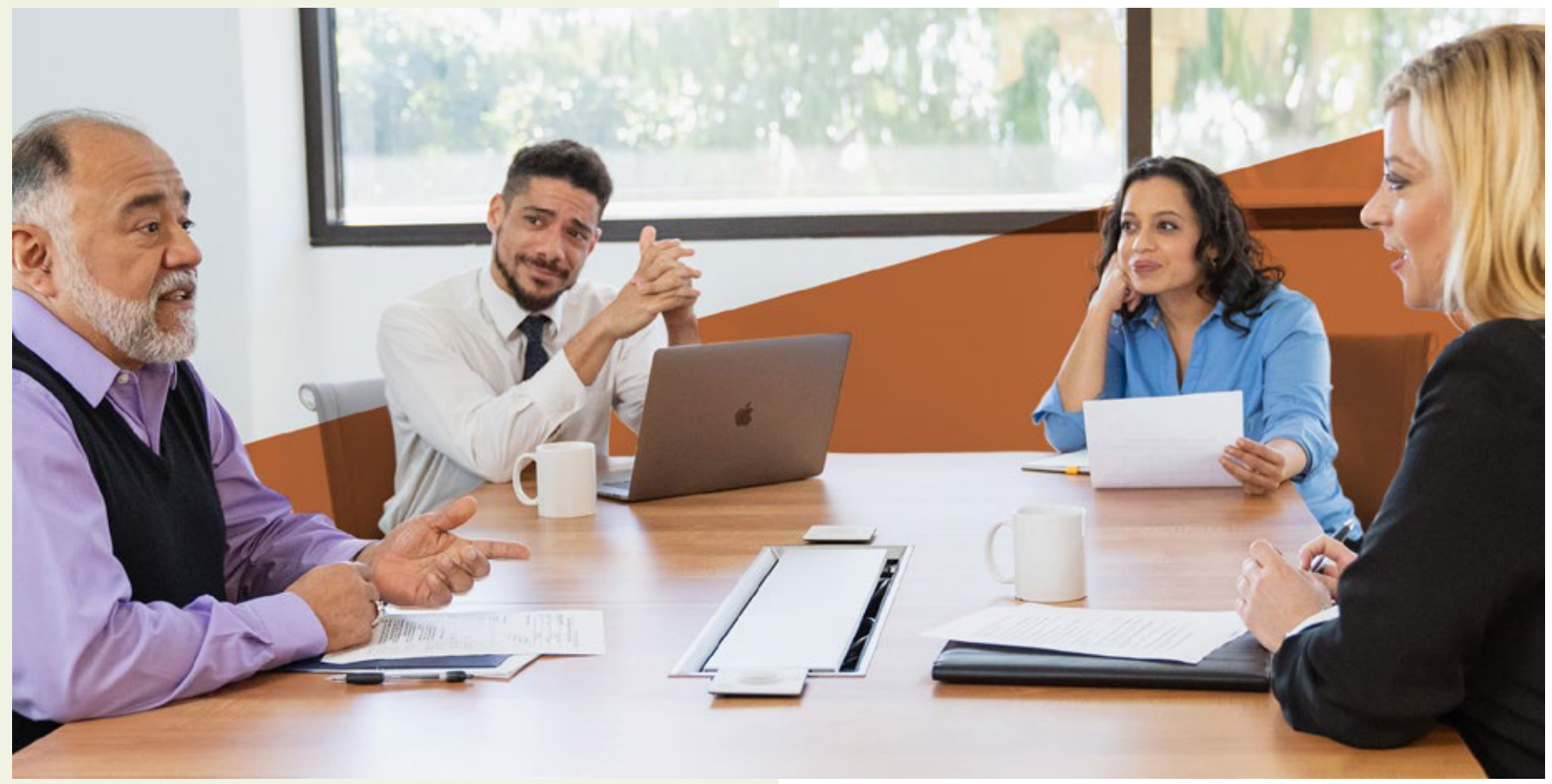


logrando como resultado un flujo de trabajo, capitalizando la experiencia, y la coordinación de los diversos profesionales del equipo de trabajo.

- Hipótesis de Segundo Grado No 3: Utilizando las prácticas aceptadas para la gestión de proyectos se puede mejorar notablemente el desempeño del equipo de trabajo a la adopción de estándares, metodologías y guías que contribuyan a aumentar el éxito y por ende disminuir el riesgo en los proyectos.

\section{Proposiciones Teóricas y Factores de Análisis}

Las proposiciones teóricas se definieron en base a los objetivos, derivadas de la teoría y de las hipótesis del caso de estudio. Los factores de análisis nacen directamente de la definición de las proposiciones de la presente investigación. La Tabla 1 muestra la relación entre proposiciones teóricas y factores de análisis.

\section{Tabla 1. Proposiciones y factores de análisis}

\begin{tabular}{|c|c|}
\hline Proposiciones & Factores de análisis \\
\hline $\begin{array}{l}\text { A. Un análisis estratégico me permite } \\
\text { determinar los elementos de } \\
\text { mejora dentro de la organización } \\
\text { para alcanzar VCS. }\end{array}$ & $\begin{array}{l}\text { 1. Análisis Estratégico } \\
\text { (Determinación de bases } \\
\text { empresariales, Análisis Externo, } \\
\text { Análisis Interno). } \\
\text { 2. Formulación Estratégica (VRIO, } \\
\text { BENCHMARKING). } \\
\text { 3. Implementación Estratégica } \\
\text { (Mapa Estratégico, Cuadro de } \\
\text { Mando, CANVAS). }\end{array}$ \\
\hline $\begin{array}{l}\text { B. Un modelo de madurez } \\
\text { proporciona el nivel de madurez } \\
\text { de la organización, además de } \\
\text { proporcionar los lineamientos base } \\
\text { para diseñar una PMO. }\end{array}$ & 1. Determinar el nivel de madurez \\
\hline $\begin{array}{l}\text { C. Diseñar una PMO permitirá } \\
\text { implementar los lineamientos } \\
\text { estratégicos de la organización en } \\
\text { los proyectos. }\end{array}$ & $\begin{array}{l}\text { 1. Determinar el tipo de PMO a } \\
\text { diseñar según el nivel de madurez. } \\
\text { 2. Definir Portafolio de la } \\
\text { organización. } \\
\text { 3. Definir Programas de la } \\
\text { organización. }\end{array}$ \\
\hline $\begin{array}{l}\text { D. La gestión del conocimiento es } \\
\text { una herramienta clave para que } \\
\text { las organizaciones consigan VCS y } \\
\text { desarrollen su capital intelectual } \\
\text { capitalizando su experiencia } \\
\text { y capturando las lecciones } \\
\text { aprendidas. }\end{array}$ & $\begin{array}{l}\text { 1. S: Sociabilización } \\
\text { 2. E: Exteriorización } \\
\text { 3. C: Combinación } \\
\text { 5. I: Internalización. }\end{array}$ \\
\hline
\end{tabular}

(Fuente: Toledo, 2017)

\section{ANÁLISIS DE LOS RESULTADOS}

El análisis de los resultados presenta los datos obtenidos en base a entrevistas, encuestas y documentación de la organización como otras fuentes de información. Primeramente, los datos se organizan en forma de tablas y gráficos para ser presentados. Posteriormente, se realiza un análisis cruzado para obtener un reporte por cada Unidad de Análisis del Caso.

Los reportes del estudio del caso tienen como finalidad entregar información consolidada, concordante y pertinente para confeccionar el reporte final del estudio empírico de la investigación. Para esto se basa en reportes divididos entre Factores de Análisis y Unidades de Análisis.

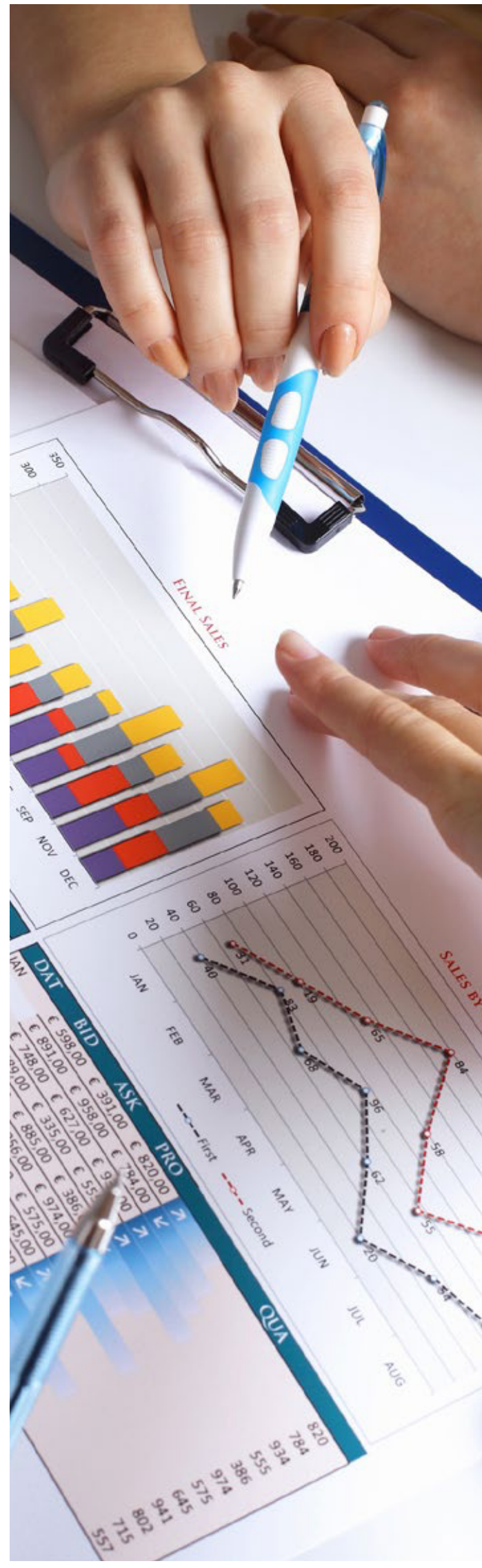




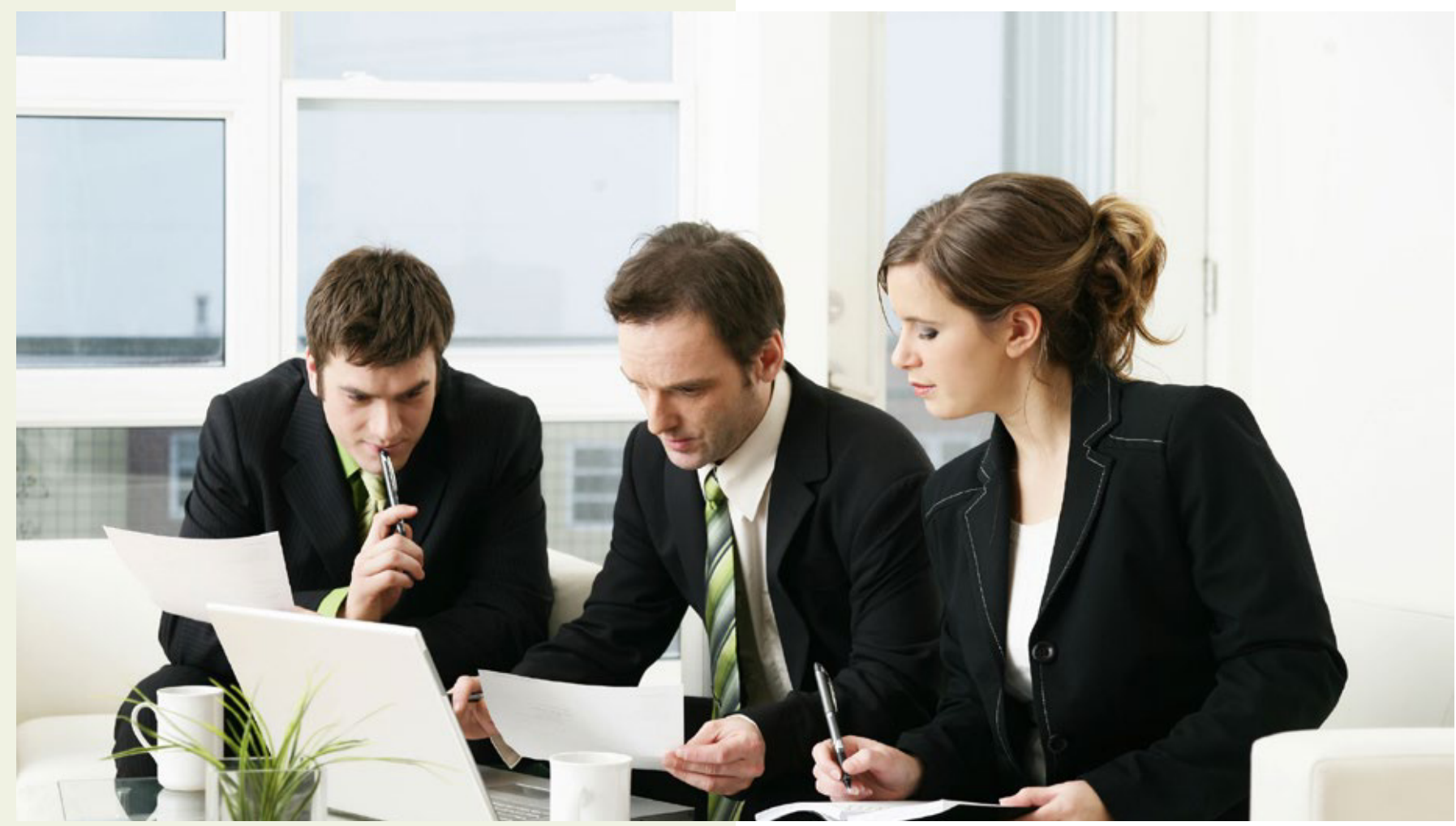

- R-01: Reporte Análisis Estratégico (Bases Empresariales, Análisis Externo, Análisis Interno).

- R-02: Reporte Formulación Estratégica (VRIO, BENCHMARKING).

- R-03: Reporte Implementación Estratégica (Mapa Estratégico, Cuadro de Mando, CANVAS).

R-04: Reporte de nivel de madurez organizacional

R-05: Reporte para determinar PMO apropiada.

R-06: Reporte de Definición de Portafolio.

R-07: Reporte de Definición de Programas.

R-08: Reporte en base a la encuesta para medir la gestión del conocimiento.

\section{Reportes de Unidades de Análisis:}

- R-09: Reporte resumen generado por encuestas y entrevistas de la Unidad de Análisis "Dirección". Se sintetiza la percepción y experiencia de la Dirección de la universidad en lo referente a las bases de la organización y el rubro, analizando la estrategia organizacional desde la visión, misión, valores y políticas, para realizar un análisis estratégico desde el punto de vista externo caracterizando los ámbitos económicos, sociales, políticos, medio ambientales, tecnológico y de mercado (EN1).

R-10: Reporte resumen generado de acuerdo a

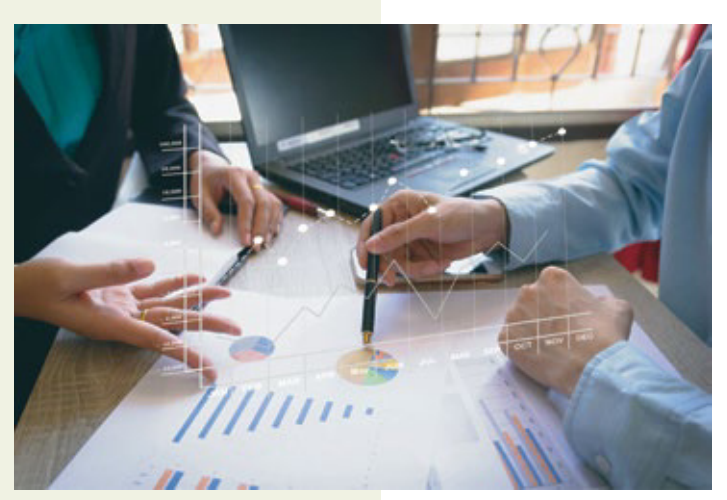

encuestas y entrevistas en la Unidad de Análisis "Sub Dirección Académica". Se enfoca en generar información valiosa para el desarrollo del análisis externo e interno, junto con la información obtenida de la Dirección Académica. Su objetivo principal es el de obtener una visión de la unidad de análisis en general y como aquella aporta en la confección del reporte final.

- R-11: Reporte resumen generado de acuerdo a encuestas aplicadas a la Unidad de Análisis "Comité de Investigación". Esta encuesta es para medir el nivel de madurez de la organización con el fin de recomendar una PMO apropiada a la realidad organizacional de la universidad, para definir portafolios y programas.

- R-12: Reporte resumen generado de la Unidad de Análisis "Académicos, la cual tiene como objetivo medir la percepción de los académicos respecto al entendimiento, aplicación e impacto de las iniciativas de gestión de conocimiento y capital intelectual, y en segunda instancia, busca medir la percepción de los académicos en la utilización de los procesos y medios de sociabilización del conocimiento en la organización.

La Figura 4, muestra el cuadro de relación de reportes, esquema que sirve de guía para la confección de la documentación de la investigación, el entregable y las conclusiones. 


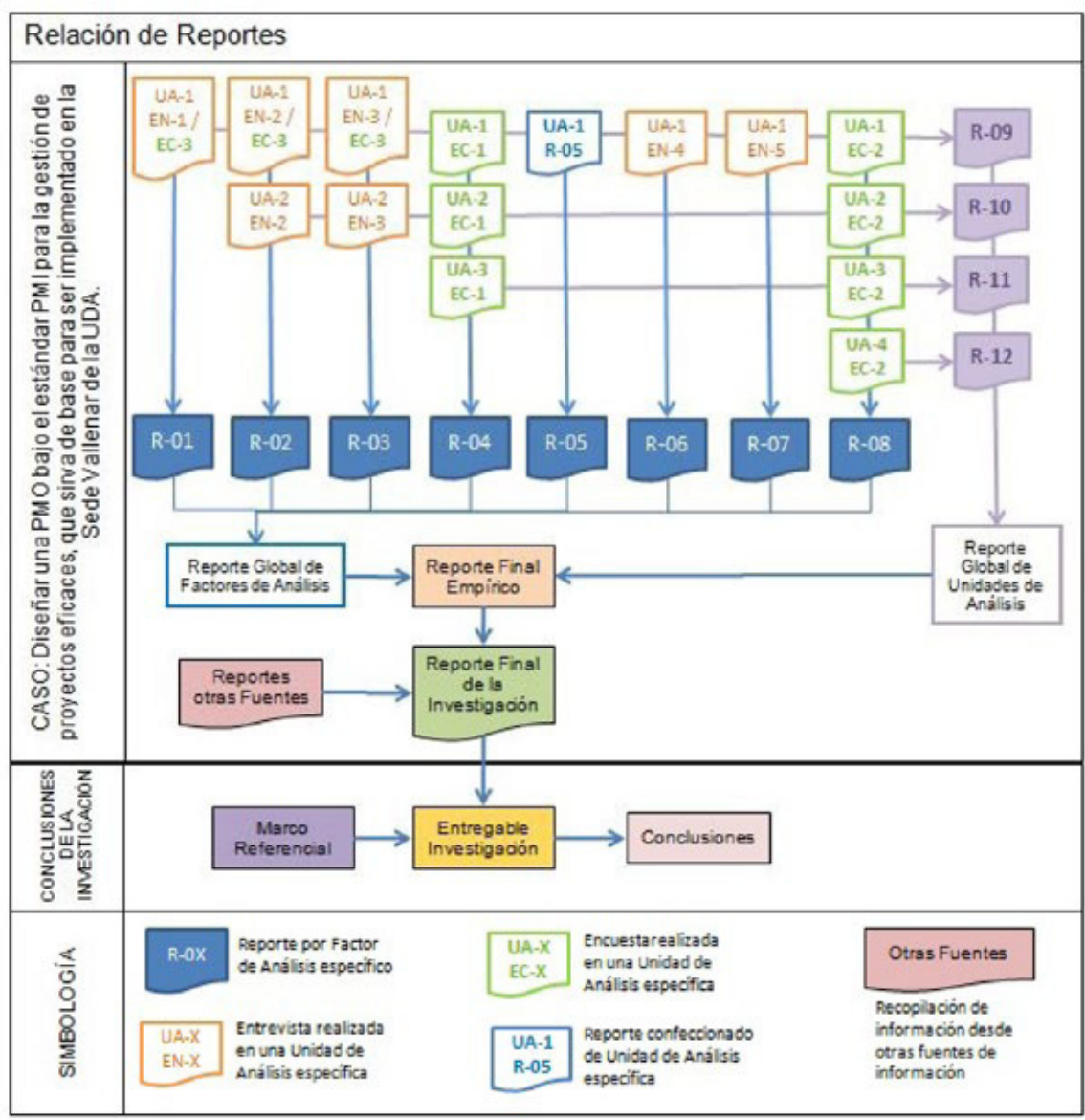

Figura 4. Relación de los reportes en la Investigación del Caso.

(Fuente: Toledo, 2017 y Alvarado, 2004)

Por su parte, la Tabla 2 muestra el cuadro de análisis empírico de la investigación, con el correspondiente cruce entre factores de análisis y unidades de análisis, y además los reportes asociados a dicha combinación analítica que forman el reporte final empírico.

Tabla 2. Cuadro de Análisis Empírico Factores de Análisis vs Unidades de Análisis.

\begin{tabular}{|c|c|c|c|c|c|c|c|c|c|}
\hline \multirow{2}{*}{$\begin{array}{l}\text { UNIDAD DE } \\
\text { ANÁLISIS }\end{array}$} & \multicolumn{8}{|c|}{ FACTORES DE ANÁLISIS } & \multirow{2}{*}{$\begin{array}{l}\text { REPORTE U.A } \\
\text { GLOBAL }\end{array}$} \\
\hline & FA.A1 & FA-A2 & FA.AB & FA-B1 & $\mathrm{FACl}$ & $\mathrm{FACL}$ & FA.C3 & FA.D1 & \\
\hline UA-1 & EN- $1 /$ EC-3 & $E N-2 / E C-3$ & $\mathrm{EN}-3 / \mathrm{EC}_{-3}$ & $\mathrm{EC}-1$ & R.05 & EN-4 & EN-5 & $\mathrm{EC}-2$ & $\bar{x}:$ UA-1: R-09 \\
\hline UA.2 & & EN-2 & EN-3 & EC-1 & & & & EC-2 & $\bar{x}: U A-2: R-10$ \\
\hline UA-3 & & & & EC-1 & & & & EC. -2 & $\bar{x}: U A-3: R-11$ \\
\hline UA.4 & & & & & & & & $E G-2$ & $\bar{x}:$ UA-4: R-12 \\
\hline $\begin{array}{l}\text { REPORTE } \\
\text { F.A GLOBAL }\end{array}$ & $\bar{x}_{F A-A L: R-01}$ & $\bar{x} F A-N: R-02$ & $\bar{X}$ FANC: $:-03$ & $\bar{X}$ FA-81:R-04 & FACC: R-05 & $\bar{x}$ FACC2:R-06 & $\bar{x}$ FACC3:R-07 & $\bar{x}$ FAADL:R-06 & $\begin{array}{c}\text { Reporte Final } \\
\text { Empirico }\end{array}$ \\
\hline
\end{tabular}
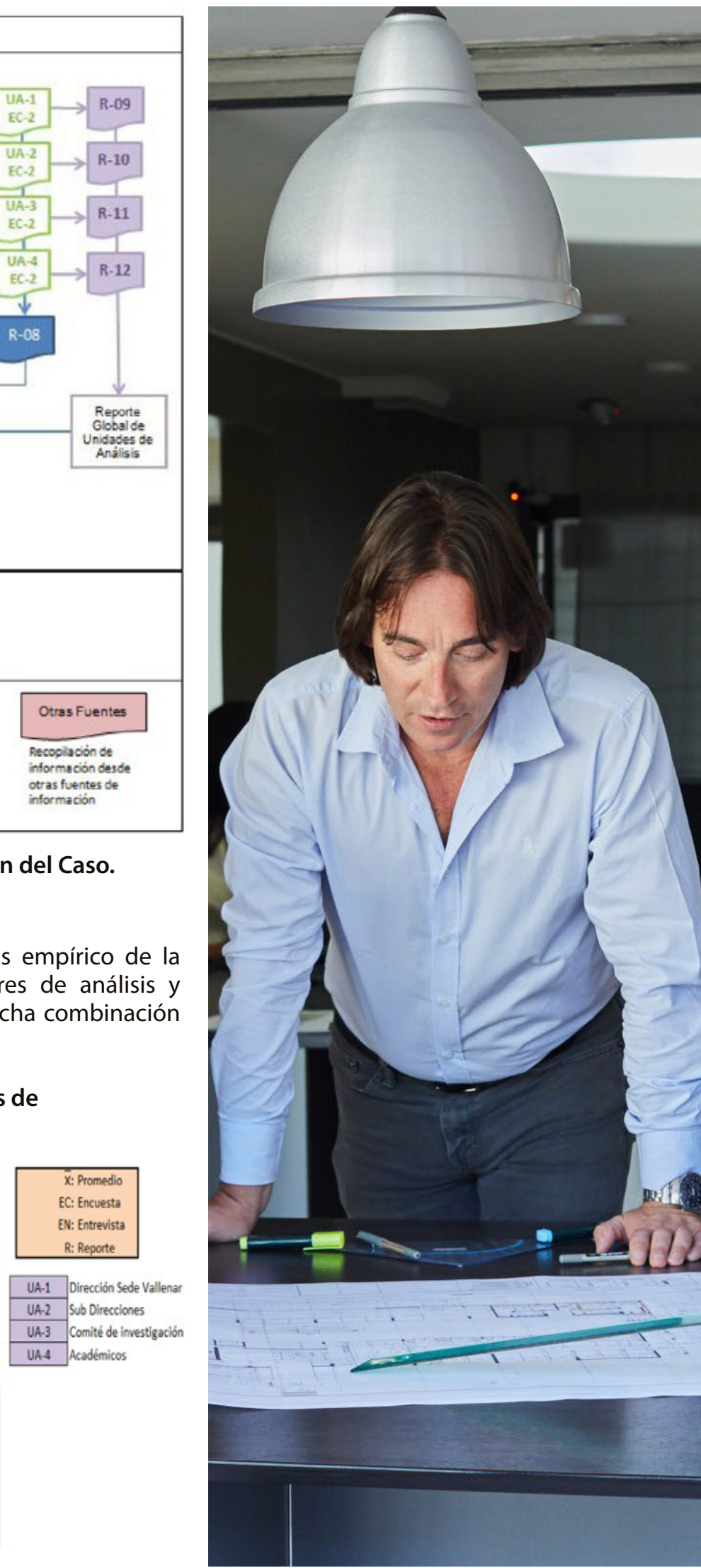

(Fuente: Toledo, 2017 y Alvarado, 2004) 
El análisis para la determinación de las bases organizacionales es fundamental para permitir sentar las bases de la adaptación del plan estratégico y posterior recomendación de PMO. En la Figura 5 se puede observar algunos de los gráficos generados en el análisis estratégico.

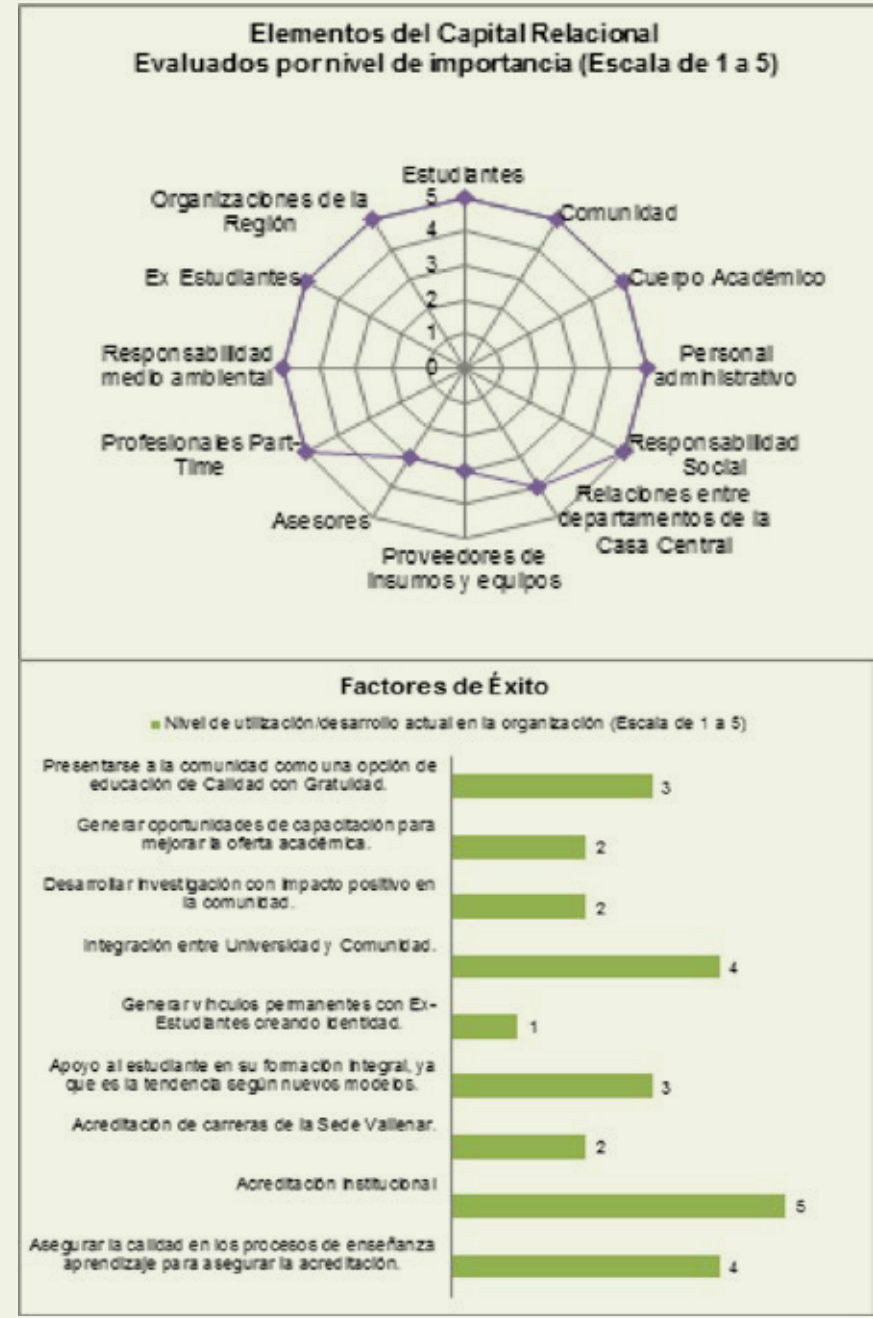

Figura 5. Gráficos del análisis estratégico (Fuente: Toledo, 2017)

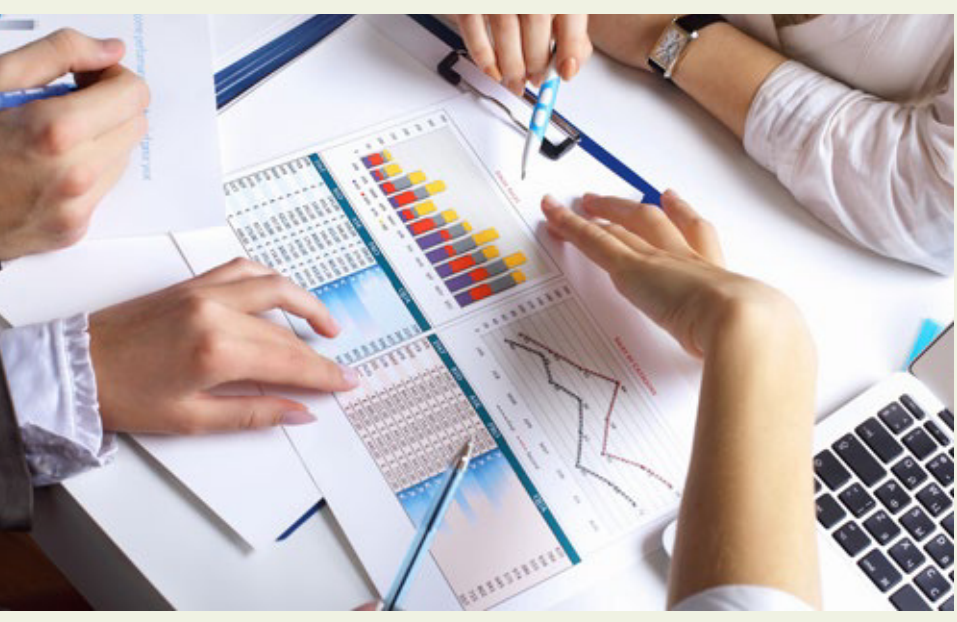

El Análisis cruzado entre las Unidades de Análisis Dirección, Sub-dirección y Comité de Investigación de la encuesta EC-3, partiendo por la estimación del nivel de madurez en gestión de proyectos para el Nivel 1 de Lenguaje Común, la cual se presenta en la Figura 6.

\section{Análisis Nivel 1: Lenguaje Común}

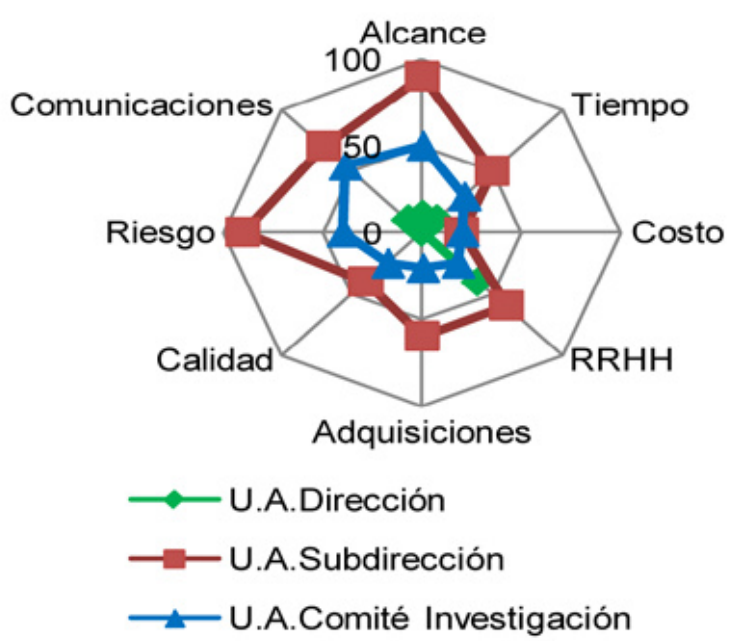

Figura 6. Gráfico de análisis cruzado de los resultados de la Encuesta para medir Nivel 1

(Fuente: Toledo, 2017)

El nivel 2 llamado procesos comunes, consta de 20 preguntas las cuales se organizan por fases del ciclo de vida según el Nivel 2 de madurez. El análisis cruzado como resultado de la estimación del nivel de madurez en gestión de proyectos para el Nivel 2 de Procesos Comunes se presenta en la Figura 7.

\section{Análisis Nivel 1: Lenguaje Común}

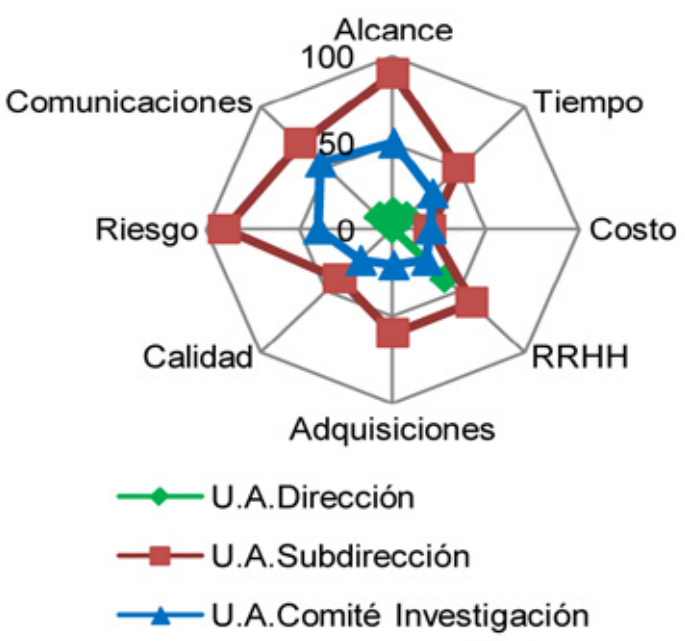

Figura 7. Gráfico de análisis cruzado de los resultados de la Encuesta para medir Nivel 2 (Fuente: Toledo, 2017) 


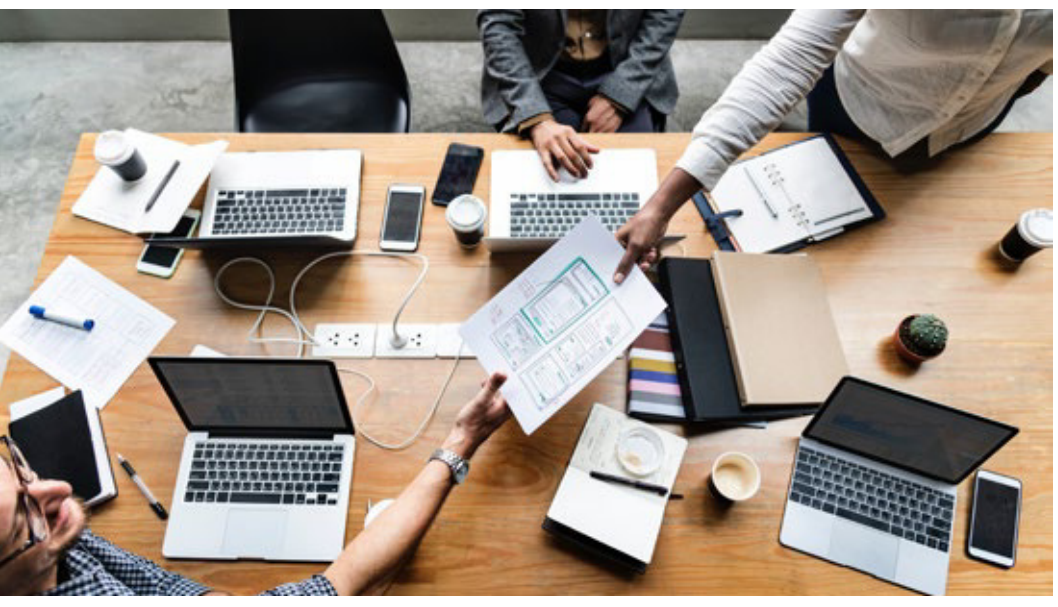

El nivel 3 por su parte, es el nivel en el que la organización reconoce que la sinergia y el control de los procesos se pueden lograr mejor mediante el desarrollo de una metodología única, como se presenta en la Figura 8.

\section{Análisis Nivel 3: Metodología Única}

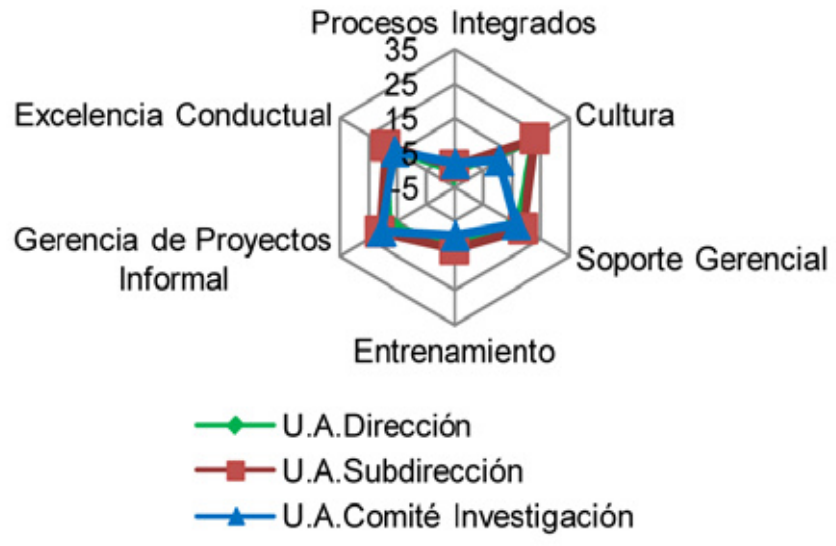

Figura 8. Gráfico de análisis cruzado de los resultados de la Encuesta para medir Nivel 3

(Fuente: Toledo, 2017)

La encuesta se divide en 3 secciones. Para cada afirmación existen cinco (5) categorías de respuesta que expresan su acuerdo o desacuerdo respecto a la situación planteada. La Tabla 3 muestra el valor numérico asignado a cada percepción.

Tabla 3. Tabla de equivalencia entre escala de percepción y escala numérica.

\begin{tabular}{l|l|c|}
\hline TED & Totalmente en desacuerdo & $-1<$ valor $<=-2$ \\
\hline EDE & En desacuerdo & $-1<$ valor $<=0$ \\
\hline NAD & Ni de acuerdo ni en desacuerdo & 0 \\
\hline DAC & De acuerdo & $0<$ valor $<=1$ \\
\hline TDA & Totalmente de acuerdo & $1<$ valor $<=2$ \\
\hline
\end{tabular}

(Fuente: Toledo, 2017)
La primera sección trata sobre la percepción de la cantidad de conocimiento generado y/o documentado en la organización. Los resultados para cada Unidad de Análisis se muestran en las Figura 9.

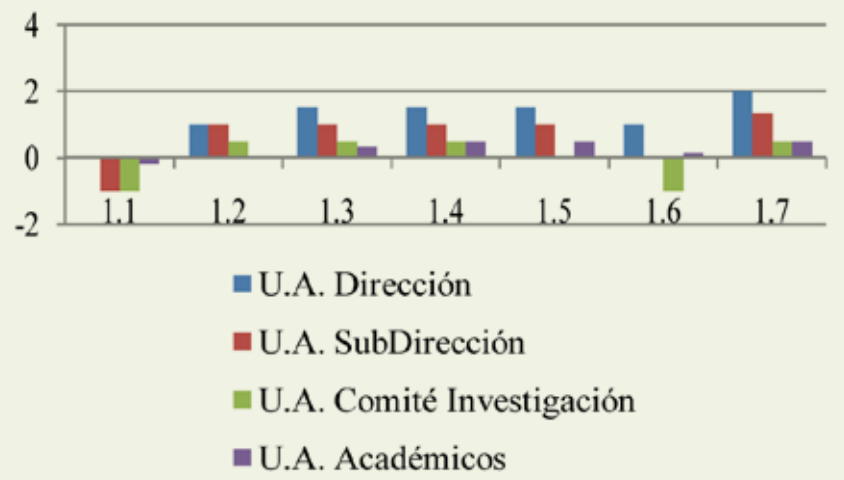

Figura 9. Análisis gráfico percepción de conocimiento generado y/o documentado en la organización.

(Fuente: Toledo, 2017)

La segunda sección trata sobre la percepción de la utilización de los procesos y medios de sociabilización del conocimiento en la organización. Los resultados para cada Unidad de Análisis se muestran en las Figura 10.

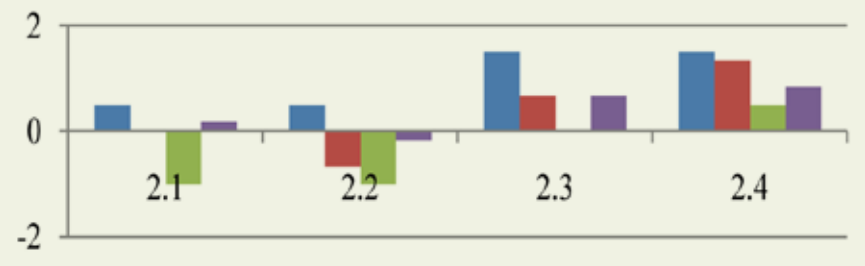
घ.A. Dirección
- U.A. SubDirección
" U.A. Comité Investigación
- U.A. Académicos

Figura 10. Análisis gráfico de la percepción de la utilización de los procesos y medios de sociabilización del conocimiento en la organización

(Fuente: Toledo, 2017)

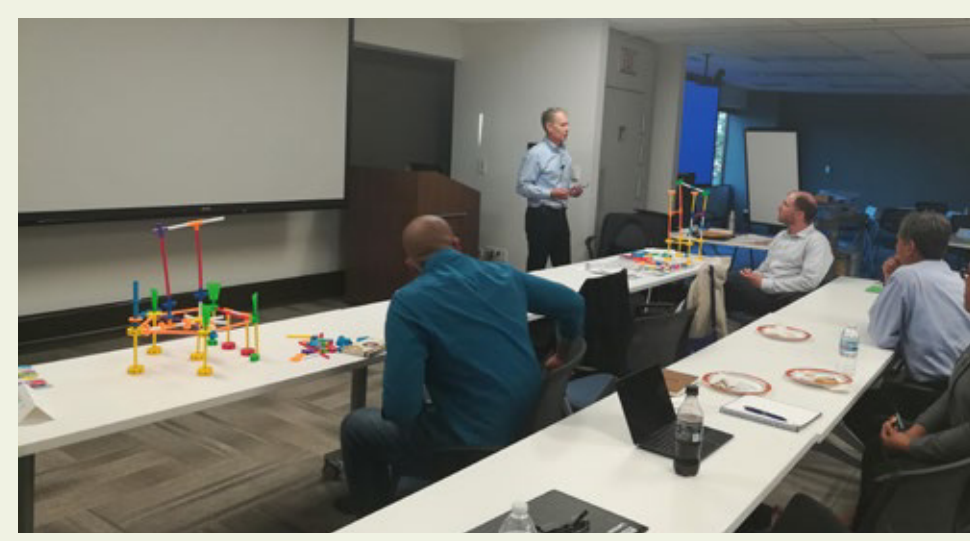


La última sección trata sobre la percepción del impacto, obteniendo como resultado de los procesos de gestión del conocimiento. Los resultados para cada Unidad de Análisis se muestran en la Figura 11.

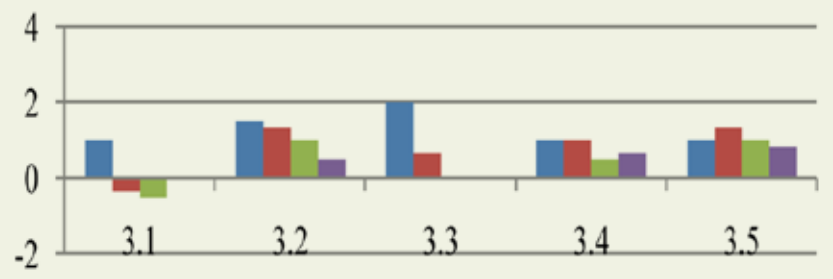

-U.A. Dirección

U.A. SubDirección

- U.A. Comité Investigación

- U.A. Académicos

Figura 11. Análisis gráfico de la percepción de los resultados de los procesos de gestión del conocimiento.

(Fuente: Toledo, 2017)

\section{ENTREGABLES DEL PROYECTO}

Se dan a conocer las recomendaciones necesarias para el diseño de la PMO, además de la entrega de los lineamientos fundamentales para un diseño de gestión de portafolios y gestión de programas. La Figura 12 muestra la estructura general del documento entregable de la investigación.

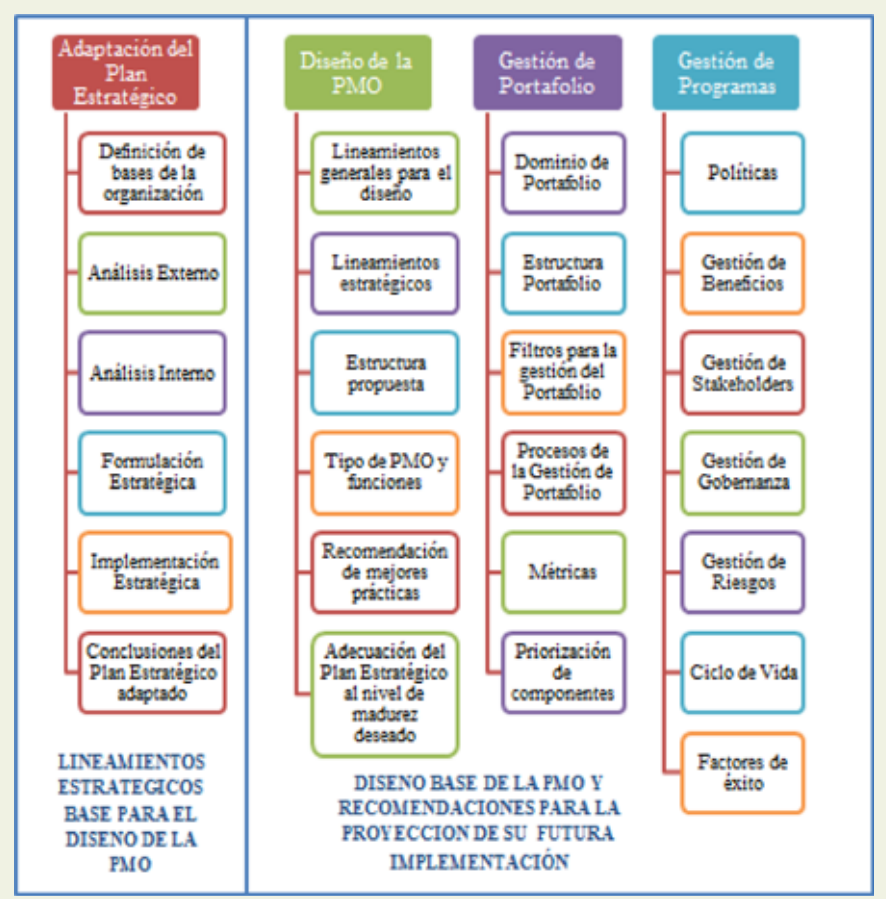

Figura 12. Esquema general del documento entregable. (Fuente: Toledo, 2017)

\section{Adaptación del Plan Estratégico}

Se expone la formulación de las bases organizaciones, el análisis externo y análisis interno llevado a cabo en la UDA Sede Vallenar, el cual luego integra la formulación estratégica anterior a través del análisis VRIO y Benchmarking estratégico, para luego pasar a la implementación estratégica de forma de mapa estratégico y cuadro de mando integral.

- Definición de las bases organizacionales: Revisión de visión, misión, valores, principales servicios que ofrece la Sede Vallenar de la Universidad de Atacama, lineamiento institucionales y principales políticas.

- Análisis externo de la organización: Análisis del entorno, análisis de los grupos estratégicos, análisis del sector y el mercado, definición de la cadena de valor de la organización, determinación de las estrategias genéricas prioritarias, definición del capital social, los modelos de excelencia organizacional seguidos en la institución y la determinación de los factores clave de éxito.

- Análisis interno de la organización: Análisis de competencias y capacidades, análisis de la cadena de valor y análisis de la gestión del conocimiento y la gestión del capital intelectual.

- Formulación estratégica: En base a los análisis anteriores, se realiza un análisis VRIO y un Benchmarking estratégico.

- Implementación estratégica: Definición de las perspectivas estratégicas, para generar un mapa estratégico de objetivos estratégicos para ser medidos y gestionados en base a un cuadro de mando integral. Finalmente se presenta un análisis bajo el modelo CANVAS.

\section{Diseño de la PMO}

En base al análisis de los resultados de la investigación, se hace evidente que aun cuando existe conciencia de la importancia de la gestión de proyectos, la organización no maneja conceptos claves relacionados con el tema, por lo cual, la organización estima iniciar un proceso de capacitación en gestión de proyectos para el equipo académico según las prácticas de PMl, con el fin de obtener el resultado esperado de manejar un lenguaje común, para luego sistemáticamente ir desarrollando oportunidades para la organización.

La recomendación del tipo de PMO apropiada depende del nivel de madurez organizacional en gestión de proyectos, la cual se mide a través de un cuestionario (Adaptado desde Kerzner, 2001 a Salmona, 2017), en dónde el encuestado debe contestar un total de 183 preguntas agrupadas en cinco temáticas específicas, donde cada temática se centran en la medición de un nivel de madurez específico. Para lograr pasar de un nivel a otro, la organización debe cumplir con un mínimo de puntaje. De no ser así, se realizan recomendaciones específicas para lograr aplicar las mejoras correspondientes y lograr pasar al siguiente nivel de madurez. De acuerdo al análisis de la 


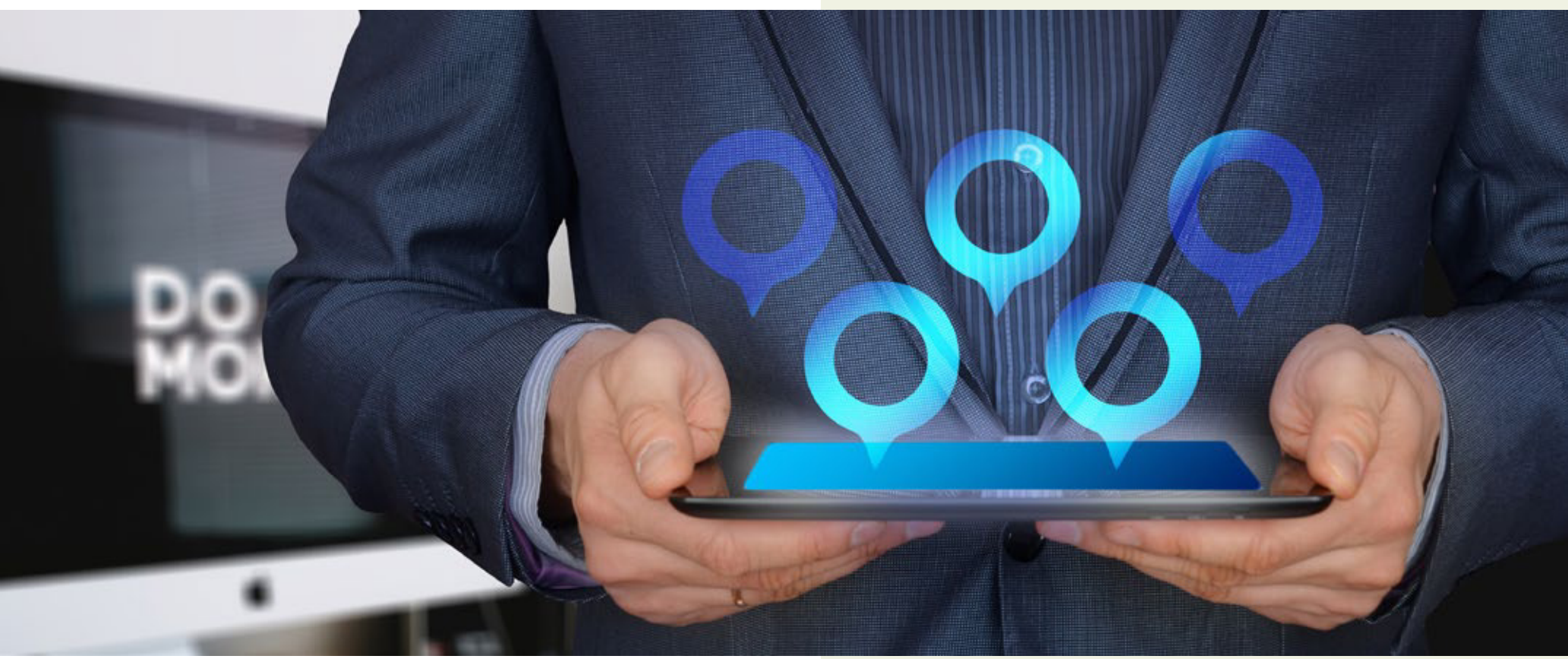

información recopilada, el investigador deduce que el nivel de madurez de la organización aún no alcanza el Nivel 1 de lenguaje común en gestión de proyectos.

- Lineamientos generales para el diseño: En términos generales, el análisis de los resultados expuestos por la encuesta para la determinó que el grado de avance de la organización tiene un nivel de madurez de Nivel menor a 1. Esto quiere decir que la UDA Sede Vallenar se encuentra en una etapa inicial de reconocimiento de la importancia de la dirección de proyectos en la perspectiva del entendimiento general de esta disciplina y del entendimiento de conceptos específicos que permiten a los miembros de la organización manejar un lenguaje común que facilita el entendimiento entre los equipo de proyecto, por lo cual se recomiendan las acciones necesarias para superar esta brecha.

- Lineamientos estratégicos: La PMO debe tener claramente definidos cuales son los objetivos en los cuales debe enfocarse. Por lo cual, en base al cuadro de mando integral propuesto y el modelo CANVAS generado, se recomienda categorizar entre objetivos estratégicos, tácticos y operativos.

- Estructura general de la propuesta: Se propone que la PMO se ubique específicamente en el área de Desarrollo Institucional y Proyectos, ya que la implementación de dicha área tendrá como fin contribuir a mejorar y fortalecer la captación de recursos corporativos para el mejoramiento de la docencia de pregrado y la investigación, mediante el aprovechamiento racional por parte de la UDA Sede Vallenar de todas las oportunidades generadoras de recursos disponibles que se presenten, utilizando como mecanismo esencial la gestión de proyectos que integren los intereses institucionales con los requerimientos de los fondos de financiamiento.
Tipo de PMO y funciones: Se recomienda que la PMO tenga la función de trabajar integradamente con el área de desarrollo institucional en el desarrollo de iniciativas alineadas a la estrategia organizacional $y$, además, ser la entidad que se encargue de apoyar al comité de investigación de la UDA Sede Vallenar. En términos generales, la PMO propuesta tiene cuatro objetivos principales, los cuales se dividen en diferentes funciones y responsabilidades:

* Apoyo y Consultoría en Gestión de Proyectos: 1.-Facilitar guías de mejores prácticas reconocidas y utilizadas en la gestión de proyectos de PMl; 2.-Unificar criterios para el manejo de un lenguaje común por parte de los equipos de proyecto, académicos y directivos.

* Capacitación en Gestión de Proyectos: 1.-Generar iniciativas para la capacitación en temas de gestión de proyectos para los académicos, funcionarios y estudiantes; 2 .-Sentar las bases para la futura elección y desarrollo de una metodología única y estándar para la gestión de los proyectos.

* Gestión de Portafolio y Programas: 1.-Alinear las iniciativas a materializarse en proyectos con los objetivos estratégicos en pos del desarrollo institucional; 2.-Seleccionar y priorizar iniciativas de proyectos que se adapten para el cumplimiento del plan de desarrollo estratégico y del plan operativo anual; 3.-Aprovechar sinergias de los proyectos con objetivos símiles, con el fin de maximizar los beneficios esperados y optimizar recursos y mejorar las oportunidades de comunicación y vinculación de los equipos de trabajo; 4.-Realizar seguimiento de los proyectos y de los resultados de los proyectos para medir beneficios prometidos.

* Gestión del Capital Intelectual: 1.-Documentar las 
leccionesaprendidasenunabasededatos, documentos estándar, herramientas y técnicas propuestas por PMI a la cual puedan acceder los equipos de proyectos; 2.-Generar planes de perfeccionamiento docente para el mejoramiento de las prácticas pedagógicas orientándolas al aprendizaje básico de gestión de proyectos por parte de los estudiantes, como una herramienta para futuros trabajos.

- Recomendaciones de mejores prácticas: Para lograr mejorar las prácticas en gestión de proyectos, se define documentación estándar a utilizar y recomendaciones para la gestión del capital intelectual en la UDA Sede Vallenar.

- Adecuación del plan estratégico al nivel de madurez organizacional: Se presenta el cuadro de mando integral y su evolución propuesta en base a las acciones estratégicas a llevar a cabo en los próximos 4 años. Cabe destacar que igualmente, se incorporarán objetivos estratégicos relacionadas a la gestión de proyectos en estas acciones, con el fin de contribuir al avance en el nivel de madurez en gestión de proyectos.

- Gestión de portafolio: El diseño de la PMO propuesta, tiene como responsabilidad principal y directa la gestión del portafolio la cual diferencie entre objetivos estratégicos, tácticos y operativos para priorizar los proyectos que tengan un impacto estratégico mayor reflejado en el cumplimiento del Plan de Desarrollo Estratégico de la UDA Sede Vallenar. En base a esto, se hace necesario definir el portafolio, lo cual permitirá lograr el alineamiento de los proyectos con la estrategia organizacional, además de planificar, implementar, generar gobernanza y maximizar el valor generado por los proyectos en la UDA Sede Vallenar. Esta gestión permite evaluar cada iniciativa respaldada por su respectivo caso de negocios propuesto, contribuyendo ya sea a generar valor y/o a asegurarlo. La gestión de portafolio es una práctica de negocios para lograr el alineamiento con la

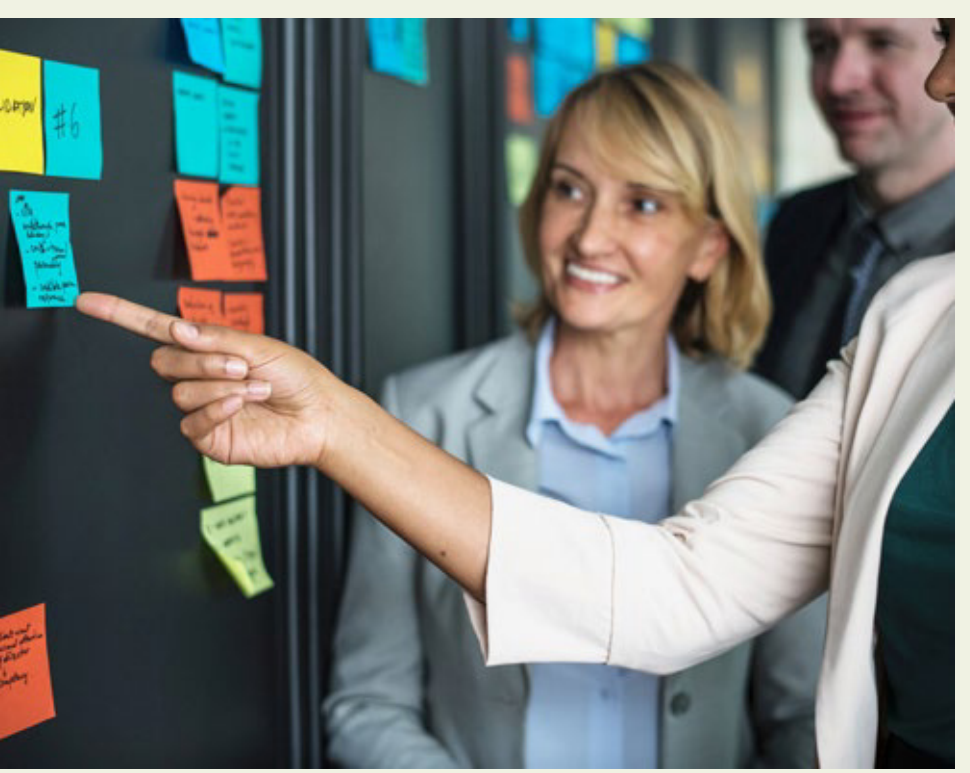

estrategia corporativa, planificar y gobernar las inversiones para maximizar el valor del negocio, manteniendo un constante seguimiento de la gestión de riesgos. La gestión de portafolio tendrá la responsabilidad de identificar, seleccionar y/o aprobar componentes propuestas para que éstas formen parte del portafolio.

- Gestión de programas: A través de la dirección de programas, las organizaciones pueden alinear múltiples proyectos para lograr la optimización o integración de costos, cronogramas, esfuerzos y beneficios. Los programas materializan las políticas institucionales las cuales son la estrategia para hacer que en cada uno de los procesos operativos y actividades diarias se realicen alineadas a los valores organizacionales. La gestión de programas debe velar por el cumplimiento de las políticas de la organización en su ejecución y en la ejecución misma del ciclo de vida del proyecto, haciendo énfasis en la gestión de comunicaciones entre stakeholders y en la maximización de beneficios lo cual está íntimamente ligado a la gestión del conocimiento. Los principales beneficios para la UDA Sede Vallenar en relación a la gestión de programas son:

* Enfocar los esfuerzos en los objetivos del negocio.

* Tener procesos formales para la identificación, gestión, realización y medición de beneficios.

* Coordinar, y realizar el seguimiento y control de los diversos proyectos que se puedan estar realizando en la organización.

* Entender de mejor forma los riesgos para su adecuada gestión.

* Realizar una adecuada gestión y control de costos.

* Enlazar la estrategia institucional, objetivos y políticas, a los proyectos para lograr el cumplimiento de la misión y visión de la organización.

- Recomendaciones: Las recomendaciones se enfocan en entender que el diseño es la guía para la futura implementación de la $\mathrm{PMO}$, la cual se recomienda realizar de manera paulatina, comenzando por la socialización de la investigación y la organización de talleres de capacitación que primeramente se enfoquen en desarrollar un léxico común en gestión de proyecto, para luego, según los lineamientos y acciones recomendadas, se puedan generar iniciativas de capacitación en temáticas más específicas de acuerdo a las inquietudes y necesidades de formación de los equipos de proyecto, hasta llegar al punto de definir una metodología única de gestión de proyectos.

\section{CONCLUSIONES}

Como conclusión respecto a la metodología empleada, se puede afirmar que es adecuada, ya que, se enfoca en el estudio de un fenómeno contemporáneo dentro de su contexto de la vida real. Los fundamentos de la investigación se apoyan en prácticas aceptadas en gestión de proyectos según PMl entregadas en su guía PMBOK. Lo anterior, se respalda en la gestión del conocimiento y los 


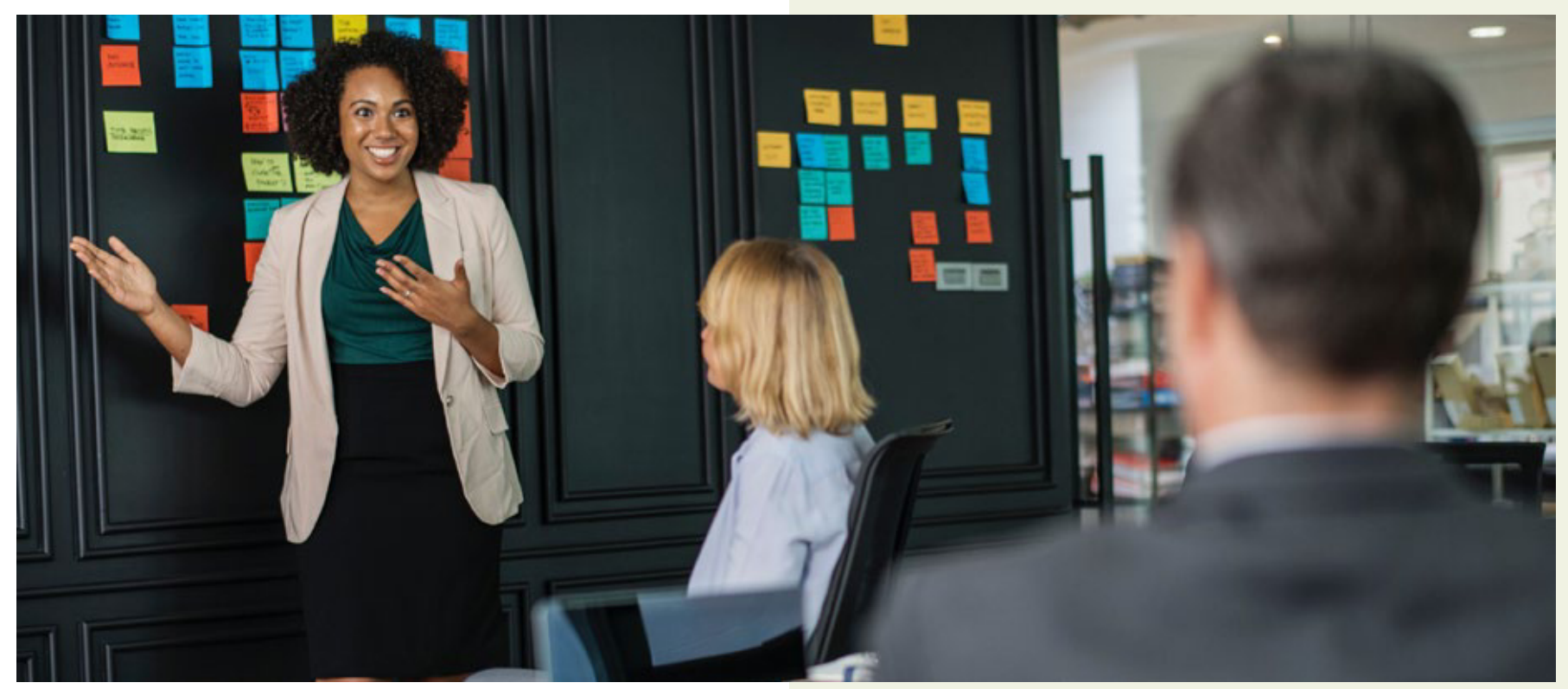

fundamentos que permiten el diseño de la PMO en forma de cuestionario para medir el nivel de madurez en gestión de proyectos.

Lo anteriormente expuesto, se justifica, ya que, la Dirección, los miembros académicos y funcionarios de la UDA Sede Vallenar no sabían la importancia del uso de guías de prácticas aceptadas para la gestión de proyectos, lo cual, fue abriendo inquietudes al desarrollarse el estudio de campo, lo cual contribuyó a identificar las principales falencias en el tema dentro de la organización.

Cabe destacar que la información obtenida desde las unidades de análisis, contribuyó de forma relevante en la definición y entendimiento de los lineamientos estratégicos y las acciones que cada uno de los miembros de la Sede Vallenar llevan a cabo, lo cual fue un elemento clave para realizar la revisión y adaptación del plan estratégico. La importancia a nivel académico radica en el desarrollo de las capacidades, habilidades y técnicas del personal docente ya sea para la investigación, la aplicación de nuevas estrategias metodológicas en aula y la participación conjunta entre docentes y estudiantes en el desarrollo de temas investigativos.

El pronóstico de las siguientes etapas en la evolución de la PMO, se enfoca en primeramente, generar la socialización del entregable y los resultados de la presente investigación patrocinada por la Dirección de la UDA Sede Vallenar y luego, lograr conseguir el apoyo mayoritario de la parte táctica y operativa igualmente. Una vez asegurado el apoyo del equipo académico, funcionarios y diversos comités se recomienda proceder con la implementación de la PMO, sus principales funciones y organizar la gestión de portafolios y la gestión de los programas que agrupen los proyectos en que su sinergia genere mayores beneficios. En paralelo, se recomienda comenzar con los talleres de capacitación en mejorar el léxico común en gestión de proyectos, implementando las iniciativas para mejorar la gestión del capital intelectual en la UDA Sede Vallenar.
Finalmente, un tema relevante e interesante de investigar puede ser la medición del impacto del diseño e implementación de la PMO en la UDA Sede Vallenar y la verificación del cumplimiento de lo comprometido como beneficio del proyecto en las operaciones cotidianas en la UDA Sede Vallenar.

\section{BIBILIOGRAFÍA}

[1] Alvarado, L. (2004). Gestión estratégica en el siglo XXI. Antofagasta, Chile: Universidad Católica del Norte

[2] Kerzner, H. (2001). Strategic planning for Project management using a project management naturity model.United States of America: Jhon Wiley \& Sons, Inc.

[3] Kerzner, H. (2003). Strategic Planning For A Project Office. Project Management Journal, 2(34), 13-25

[4] Nonaka, I., \& Takeuchi, H. (1995). The Knowledge-creating Company: How Japanese Companies Create the Dynamics of Innovation. (O. U. Press, Ed.)

[5] Porter, M. (1985). The Competitive Advantage: Creating and Sustaininng Superior Performance. NY: Free Press.

[6] Project Management Institute. (2017). Fundamentos para la dirección de proyectos (Guía PMBOK).

[7] Newtown Square, Pensilvania EE.UU: Project Management Institute, Inc.

[8] Schlichter, J. (1999). Surveying Project Management Capabilities. PM Network, 4(13), 39-40.

[9] Shaw, E. (1999). A guide to the qualitative research process: evidence from a small firm study. Qualitative Market Research: An International Journal, 2(2), 59-70

[10] Toledo Perez de Arce, E. (2017). Diseño de una PMO basada en el estándar PMI para la gestión de proyectos en la sede Vallenar de la Universidad de Atacama, Chile. (Tesis para optar al grado de Magister en Gestión Integral de Proyectos). Universidad Católica del Norte.

[11] Universidad de Atacama. (2007). Modelo Educativo de la Universidad de Atacama. Copiapó.

[12] Universidad de Atacama Dirección de Planificación. (2014). Plan de Desarrollo Estratégico 2015-2019. Copiapó.

[13] Yin, R. K. (1994). Case study research: design and methods. Thousand Oaks, London, New Delhi : Sage Publications, 1994.

[14] Yin, R. K. (2003). Case Study Research: Design and Methods (Third Edition ed.). Sage, 2003 